\title{
A New Family of Integrable Extended Multi-band Hubbard Hamiltonians]
}

\author{
J.Ambjorn" \\ Niels Bohr Institute \\ Blegdamsvej 17, Copenhagen, Denmark

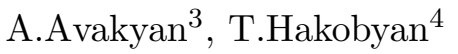 \\ Yerevan Physics Institute, \\ Br.Alikhanian st.2, 375036, Yerevan, Armenia \\ A.Sedrakyan呵, \\ Niels Bohr Institute \\ Blegdamsvej 17, Copenhagen, Denmark
}

January 1997

\begin{abstract}
We consider exactly solvable $1 d$ multi-band fermionic Hamiltonians, which have affine quantum group symmetry for all values of the deformation. The simplest Hamiltonian is a multi-band $t-J$ model with vanishing spin-spin interaction, which is the affinization of an underlying $X X Z$ model. We also find a multi-band generalization of standard $t-J$ model Hamiltonian.
\end{abstract}

\footnotetext{
${ }^{1}$ This work was supported in part by INTAS grant $94-840$

${ }^{2}$ e-mail:ambjorn@nbivms.nbi.dk

3 e-mail:avakyan@lx2.yerphi.am

${ }^{4}$ e-mail:hakob@lx2.yerphi.am

${ }^{5}$ e-mail:sedrak@nbivms.nbi.dk; permanent address: Yerevan Physics Institute
} 


\section{Introduction}

In [1] we developed the technique for construction of spin chain Hamiltonians, which have the energy levels of finite $X X Z$ Heisenberg magnets with a degeneracy of levels due to an affine quantum group symmetry. One can call this procedure an affinization of $X X Z$ model. The first example of this type model was constructed in [2], giving rise to the Hubbard Hamiltonian in the infinite repulsion limit. In [3] we have fermionized the simplest examples of this newly defined family of spin chain models and have shown that it leads to extensions of one-band Hubbard Hamiltonians. The $\eta$-pairing mechanism introduced by Yang [4, 5] was found in one of the examples, in addition to other extended exactly solvable Hubbard models with super-conducting ground state [6, 7, 8, 9, 10].

An interesting aspect of these models is that the existence of an affine quantum group symmetry and the associated degeneracy of levels might lead to a new type of string theories.

In this article we extend the investigations to $t-J$ models initiated in [11, 12]. In sec.2 we review the construction of spin chain Hamiltonians which possesses affine quantum group symmetry [1, 3]. In sec.3 we use this construction to obtain a multi-band $t-J$ model at $J=0$ which is the affinization of the $X X Z$ Heisenberg Hamiltonian. In sec. 4 we show how a multi-band $t-J$ model is obtained from the standard one-band $t-J$ model by the action of the affine quantum group. It is shown that some simple multi-band extension of the $t-J$ model, where the spin-spin coupling term consists of the interaction between the total spins, i.e. the sum of the spins of all bands, at nearest neighbor sites, contains an affine quantum group symmetry.

\section{Quantum group invariant Hamiltonians for reducible rep- resentations}

Let $\mathbf{V}=\oplus_{i=1}^{N} \mathbf{V}_{\lambda_{i}}$ be a direct sum of finite dimensional irreducible representations $\mathbf{V}_{\lambda_{i}}$ of quantum group $U_{q} g$ [13, 14, 15]. We denote by $\mathbf{V}\left(x_{1}, \ldots, x_{N}\right)$ the representation with spectral parameters $x_{i}$ of the corresponding affine quantum group $U_{q} \hat{g}$ [15]:

$$
\mathbf{V}\left(x_{1}, \ldots, x_{N}\right)=\bigoplus_{i=1}^{M} \mathbf{N}_{\lambda_{i}} \hat{\otimes} \mathbf{V}_{\lambda_{i}}\left(x_{i}\right)
$$

where all the $\mathbf{V}_{\lambda_{i}}\left(x_{i}\right)$ are $M$ nonequivalent irreps and $\mathbf{N}_{\lambda_{i}} \simeq \mathbf{C}^{N_{i}}$ have dimensions equal to the multiplicity of $\mathbf{V}_{\lambda_{i}}\left(x_{i}\right)$ in $\mathbf{V}\left(x_{1}, \ldots, x_{N}\right)$. Note that $\sum_{i=1}^{M} N_{i}=N$. The ${ }^{\wedge}$ over the tensor product signifies that $U_{q} \hat{g}$ does not act on $\mathbf{N}_{\lambda_{i}} \hat{\otimes} \mathbf{V}_{\lambda_{i}}\left(x_{i}\right)$ by means of comultiplication $\Delta$ but instead acts as id $\otimes g$.

In [3] the general matrix form of intertwining operator

$$
\begin{aligned}
& H\left(x_{1}, \ldots, x_{N}\right): \\
& \quad \mathbf{V}\left(x_{1}, \ldots, x_{N}\right) \otimes \mathbf{V}\left(x_{1}, \ldots, x_{N}\right) \rightarrow \mathbf{V}\left(x_{1}, \ldots, x_{N}\right) \otimes \mathbf{V}\left(x_{1}, \ldots, x_{N}\right), \\
& \quad\left[H\left(x_{1}, \ldots, x_{N}\right), \Delta(a)\right]=0, \quad \forall a \in U_{q} \hat{g}
\end{aligned}
$$

had been written using the projection operators

$$
X_{b}^{a}=|a\rangle\langle b|
$$

Here the vectors $|a\rangle$ span the space $\mathbf{V}$. In accordance with the decomposition (1) we will use the double index $a=\left(n_{i}, a_{i}\right), i=1, \ldots, M$ where the first index $n_{i}=1, \ldots, N_{i}$ characterizes 
the multiplicity of $\mathbf{V}_{\lambda_{i}}$ and the second one $a_{i}=1, \ldots, \operatorname{dim} \mathbf{V}_{\lambda_{i}}$ is the vector index of $\mathbf{V}_{\lambda_{i}}$. Then the intertwining operator (2) is

$$
\begin{array}{r}
H(A, B)=\sum_{i, j=1}^{M}\left(\sum_{n_{i}, n_{j}, m_{i}, m_{j}} A_{i j n_{i} n_{j}}^{m_{i} m_{j}} \sum_{a_{i}, a_{j}} X_{\left(m_{i}, a_{i}\right)}^{\left(n_{i}, a_{i}\right)} \otimes X_{\left(m_{j}, a_{j}\right)}^{\left(n_{j}, a_{j}\right)}\right. \\
\left.+\sum_{n_{i}, n_{j}, m_{i}, m_{j}} B_{i j n_{i} n_{j}}^{m_{i} m_{j}} \sum_{a_{i}, a_{j}, a_{i}^{\prime}, a_{j}^{\prime}} R_{i j}{ }_{i j}^{a_{i}^{\prime} a_{j}}\left(x_{i} a_{j}^{\prime} / x_{j}\right) X_{\left(m_{i}, a_{i}\right)}^{\left(n_{j}, a_{j}^{\prime}\right)} \otimes X_{\left(m_{j}, a_{j}\right)}^{\left(n_{i}, a_{i}^{\prime}\right)}\right),
\end{array}
$$

where the $R$-matrix

$$
R_{\mathbf{V}_{\lambda_{i}} \otimes \mathbf{V}_{\lambda_{j}}}\left(x_{i} / x_{j}\right)\left|a_{i}\right\rangle \otimes\left|a_{j}\right\rangle=\sum_{a_{i}^{\prime}, a_{j}^{\prime}} R_{i j}^{a_{i}^{\prime} a_{i}^{\prime} a_{j}^{\prime}}\left(x_{i} / x_{j}\right)\left|a_{i}^{\prime}\right\rangle \otimes\left|a_{j}^{\prime}\right\rangle
$$

is the intertwining operator between two actions of affine quantum group $U_{q} \hat{g}$ on $\mathbf{V}_{\lambda_{i}} \otimes \mathbf{V}_{\lambda_{j}}$, which are induced correspondingly by comultiplication $\Delta$ and opposite comultiplication $\bar{\Delta}$ [13, 14, 15]:

$$
R_{\mathbf{V}_{\lambda_{i}} \otimes \mathbf{V}_{\lambda_{j}}}\left(x_{i} / x_{j}\right) \Delta(g)=\bar{\Delta}(g) R_{\mathbf{V}_{\lambda_{i}} \otimes \mathbf{V}_{\lambda_{j}}}\left(x_{i} / x_{j}\right) .
$$

$A_{i j}$ and $B_{i j}, B_{i i}=0$ in (顿) are arbitrary matrices. In general, $H(A, B)$ depends on deformation parameter $q$ of quantum group, which is included in the $R$-matrix. Note that $R_{\mathbf{V}_{\lambda_{i}} \otimes \mathbf{V}_{\lambda_{j}}}\left(x_{i} / x_{j}\right)$ does not depend on $q$ and is identity only if $\lambda_{i}$ or $\lambda_{j}$ are trivial onedimensional representations. So, in the special case, when the only nontrivial $R$-matrixes in (4) are between representations, one of which is trivial representation, the expression of $H(A, B)$ doesn't depend on $q$. Then $H(A, B)$ commutes with the quantum group action for all values of deformation parameter. In the following we consider only this case.

Following [2, 1] we can from the operator $H$ construct the following Hamiltonian acting on $\mathbf{W}=\mathbf{V}^{\otimes L}:$ 6

$$
\mathcal{H}=\sum_{i=1}^{L-1} H_{i i+1},
$$

where the indices $i$ and $i+1$ denote the sites where $H$ acts non-trivially. By the construction, $\mathcal{H}$ is quantum group invariant:

$$
\left[\mathcal{H}, \Delta^{L-1}(g)\right]=0, \quad \forall g \in U_{q} \hat{g}
$$

Let us define the projection operators $\mathcal{Q}^{i}$ on $\mathbf{V}$ for each class of equivalent irreps $\left(\lambda_{i}, x_{i}\right)$, $i=1, \ldots, M$

$$
\begin{gathered}
\mathcal{Q}^{i} v_{j}=\delta_{i j} v_{j}, \quad \forall v_{j} \in V_{\lambda_{j}}\left(x_{j}\right) \\
\sum_{i=1}^{M} \mathcal{Q}^{i}=\mathrm{id}, \quad\left(\mathcal{Q}^{i}\right)^{2}=\mathcal{Q}^{i}
\end{gathered}
$$

Their action on $\mathbf{W}$ is given by

$$
\mathcal{Q}^{i}=\sum_{l=1}^{L} \mathcal{Q}_{l}^{i}
$$

\footnotetext{
${ }^{6}$ Here and in the following we omit the dependence on $x_{i}$
} 
It is easy to see that these projections commute with Hamiltonian $\mathcal{H}$ and quantum group $U_{q} \hat{g}$ :

$$
\left[\mathcal{Q}^{i}, \mathcal{H}\right]=0, \quad\left[\mathcal{Q}^{i}, U_{q} \hat{g}\right]=0
$$

Denote by $\mathbf{W}_{p_{1} \ldots p_{M}}$ the subspace of $\mathbf{W}$ with values $p_{i}$ of $\mathcal{Q}^{i}$ on it. Then we have the decomposition

$$
\mathbf{W}=\bigoplus_{\substack{p_{1}, \ldots, p_{M} \\ p_{1}+\ldots+p_{M}=L}} \mathbf{W}_{p_{1} \ldots p_{M}}
$$

Let $\mathbf{V}^{0}$ be the linear space, spanned by the highest weight vectors in $V$ :

$$
\mathbf{V}^{0}:=\oplus_{i=1}^{N} v_{\lambda_{i}}^{0}
$$

where $v_{\lambda_{i}}^{0} \in \mathbf{V}_{\lambda_{i}}$ is a highest weight vector. We also define $\mathbf{W}^{0}:=\mathbf{V}^{0} \otimes L$. The space $\mathbf{W}^{0}$ is $\mathcal{H}$-invariant. For general $q$ the action of $U_{q} \hat{g}$ on $\mathbf{W}^{0}$ generate whole space $\mathbf{W}$. Indeed, the $U_{q} \hat{g}$-action on each state of type $v_{\lambda_{i_{1}}}^{0} \otimes \ldots \otimes v_{\lambda_{i_{L}}}^{0}$ generates the space $\mathbf{V}_{\lambda_{i_{1}}} \otimes \ldots \otimes \mathbf{V}_{\lambda_{i_{L}}}$, because the tensor product of finite dimensional irreducible representations of an affine quantum group is irreducible [16].

Consider now the subspace $\mathbf{W}_{p_{1} \ldots p_{M}}^{0}=\mathbf{W}^{0} \cap \mathbf{W}_{p_{1} \ldots p_{M}}$. According to (đ) we have the decomposition

$$
\mathbf{W}^{0}=\bigoplus_{\substack{p_{1}, \ldots, p_{M} \\ p_{1}+\ldots+p_{M}=L}} \mathbf{W}_{p_{1} \ldots p_{M}}^{0}
$$

Note that

$$
d_{p_{1} \ldots p_{M}}:=\operatorname{dim} \mathbf{W}_{p_{1} \ldots p_{M}}^{0}=\left(\begin{array}{c}
L \\
p_{1} \ldots p_{M}
\end{array}\right) N_{1}^{p_{1}} \ldots N_{M}^{p_{M}} .
$$

Let us define by $\mathcal{H}_{0}$ the restriction of $\mathcal{H}$ on $\mathbf{W}_{0}: \mathcal{H}_{0}:=\left.\mathcal{H}\right|_{\mathbf{W}_{0}}$. It follows from (6) that Hamiltonians $\mathcal{H}$ and $\mathcal{H}_{0}$ have block diagonal form with respect to the decompositions (7) and (8), respectively. Every eigenvector $w_{\alpha_{p_{1} \ldots p_{M}}}^{0} \in \mathbf{W}_{p_{1} \ldots p_{M}}^{0}$ with energy value $E_{\alpha_{p_{1} \ldots p_{M}}}$ gives rise to an irreducible $U_{q} \hat{g}$-multiplet $\mathbf{W}_{\alpha_{p_{1} \ldots p_{M}}}$ of dimension

$$
\operatorname{dim} \mathbf{W}_{\alpha_{p_{1} \ldots p_{M}}}=\prod_{k=1}^{M}\left(\operatorname{dim} \mathbf{V}_{\lambda_{k}}\right)^{p_{k}}
$$

On $\mathbf{W}_{\alpha_{p_{1} \ldots p_{M}}}$ the Hamiltonian $\mathcal{H}$ is diagonal with eigenvalue $E_{\alpha_{p_{1} \ldots p_{M}}}$. In particular, in the case when all $\mathbf{V}_{\lambda_{i}}$ are equivalent, the degeneracy levels are the same for all $E_{\alpha_{p_{1} \ldots p_{M}}}$ and are equal to $\left(\operatorname{dim} \mathbf{V}_{\lambda}\right)^{L}$.

Now, let us assume we know the energy spectrum $E_{\alpha_{p_{1} \ldots p_{M}}}$ for $\mathcal{H}_{0}$. Then the statistical sum is given by

$$
Z_{\mathcal{H}_{0}}(\beta)=\sum_{\substack{p_{1}, \ldots, p_{M} \\ p_{1}+\ldots+p_{M}=L}} \sum_{\alpha_{p_{1} \ldots p_{M}}=1}^{d_{p_{1} \ldots p_{M}}} \exp \left(\beta E_{\alpha_{p_{1} \ldots p_{M}}}\right),
$$

and it follows that the statistical sum of $\mathcal{H}$ has the following form:

$$
Z_{\mathcal{H}}(\beta)=\sum_{\substack{p_{1}, \ldots, p_{M} \\ p_{1}+\ldots+p_{M}=L}} \prod_{k=1}^{M}\left(\operatorname{dim} \mathbf{V}_{\lambda_{k}}\right)^{p_{k}} \sum_{\alpha_{p_{1} \ldots p_{M}}=1}^{d_{p_{1} \ldots p_{M}}} \exp \left(\beta E_{\alpha_{p_{1} \ldots p_{M}}}\right)
$$

So, if the underlying Hamiltonian $\mathcal{H}_{0}$ is integrable and its eigenvectors and eigenvalues can be found, then we know these for $\mathcal{H}$ too. Acting with the quantum group on all eigenvectors of an energy level of $\mathcal{H}_{0}$ one obtains the whole eigenspace of $\mathcal{H}$ for this level. 


\section{Multi-band $t-J$ model with vanishing spin-spin coupling $J=0$}

Let us consider here the quantum group $U_{q} \widehat{s l}_{2}$. We choose $\mathbf{V}=\mathbf{V}_{0} \oplus \mathbf{V}_{j}$ for decomposition (11), i.e. we take a direct sum of the trivial spin-0 and the $2 j+1$-dimensional spin- $j$ representation of $U_{q} s l_{2}$. The $R$-matrix in the second term in (伍) does not depend on $q$ and spectral parameters $x_{i}$ and coincides with the identity, as it was mentioned above. So, using (4) and (5), we obtain the following Hamiltonian

$$
\begin{array}{r}
\mathcal{H}\left(t, V_{1}, V_{2}\right)=\sum_{i=1}^{L-1}\left[-t \sum_{p=1}^{2 j+1}\left(X_{i 0}^{p} X_{i+1}^{0}+X_{i+10}^{p} X_{i p}^{0}\right)+V_{1} X_{i 0}^{0} X_{i+1}^{0}\right. \\
\left.+V_{2} \sum_{p, p^{\prime}=1}^{2 j+1} X_{i p}^{p} X_{i+1} p_{p^{\prime}}\right]
\end{array}
$$

The Hamiltonian $\mathcal{H}=\sum_{i} H_{i i+1}$ was constructed from the operator $H=H_{i i+1}$, where $H$ can be written in the matrix form

$$
H=\left(\begin{array}{cccc}
V_{1} & 0 & 0 & 0 \\
0 & 0 & -t \cdot \mathrm{id} & 0 \\
0 & -t \cdot \mathrm{id} & 0 & 0 \\
0 & 0 & 0 & V_{2} \cdot \mathrm{id}
\end{array}\right) .
$$

The projection on the highest weight space coincides with the constructing block of the $X X Z$ Hamiltonian in an external magnetic field. This implies that the restriction of (12) to the space $\mathbf{W}^{0}$ is

$$
\begin{aligned}
\mathcal{H}_{0}\left(t, W_{1}, W_{2}\right) & =\mathcal{H}_{X X Z}(t, \Delta, B) \\
& =-\frac{t}{2} \sum_{i=1}^{L-1}\left(\sigma_{i}^{x} \sigma_{i+1}^{x}+\sigma_{i}^{y} \sigma_{i+1}^{y}+\Delta \sigma_{i}^{z} \sigma_{i+1}^{z}+\frac{B}{2} \sigma_{i}^{z}\right),
\end{aligned}
$$

where

$$
\Delta=-\frac{V_{1}+V_{2}}{2 t}, \quad B=\frac{2}{t}\left(V_{1}-V_{2}\right)
$$

For the special case $V_{1}+V_{2}=0 \mathcal{H}_{0}$ gives rise to the free fermionic (or equivalently $X Y$ ) Hamiltonian $(\Delta=0)$.

The projection operators $X_{b}^{a}$ are expressed through the fermionic creation-annihilation operators as follows

$$
\begin{gathered}
X_{i 0}^{p}=\mathcal{P} c_{i, p}^{+}, \\
X_{i p}^{0}=c_{i, p} \mathcal{P}, \\
X_{i p}^{p}=n_{i, p} \mathcal{P}=\mathcal{P} n_{i, p}, \quad X_{i 0}^{0}=\left(1-n_{i}\right) \mathcal{P}=\mathcal{P}\left(1-n_{i}\right)
\end{gathered}
$$

Here we introduced the projection operator which forbids double occupation on all sites

$$
\mathcal{P}=\prod_{i=1}^{L} \mathcal{P}_{i}, \quad \mathcal{P}_{i}=\prod_{p \neq p^{\prime}}\left(1-n_{i, p} n_{i, p^{\prime}}\right)
$$

and the total particle number $n_{i}=\sum_{p} n_{i, p}$ at site $i$. 
After the substitution of the fermionic representation (16) into (12) we obtain

$$
\begin{array}{r}
\mathcal{H}\left(t, V_{1}, V_{2}\right)=\mathcal{P} \sum_{i=1}^{L-1}\left[-t \sum_{p=1}^{2 j+1}\left(c_{i, p}^{+} c_{i+1, p}+c_{i+1, p}^{+} c_{i, p}\right)+\right. \\
\left.V n_{i} n_{i+1}-V_{1}\left(n_{i}+n_{i+1}\right)+V_{1}\right] \mathcal{P},
\end{array}
$$

where $V=V_{1}+V_{2}$. The chemical potential term $-V_{1} \sum_{i=1}^{L-1}\left(n_{i}+n_{i+1}\right)$ commutes with $\mathcal{H}$ and can be omitted. So, up to unessential boundary and constant terms (17) is a multicomponent $t-J$ model with vanishing spin-spin coupling $(J=0)$

$$
\mathcal{H}(t, V)=\sum_{i=1}^{L-1}\left[-t \sum_{p=1}^{2 j+1}\left(c_{i, p}^{+} c_{i+1, p}+c_{i+1, p}^{+} c_{i, p}\right)+V n_{i} n_{i+1}\right]+\sum_{i=1}^{L} \sum_{\substack{p \neq p^{\prime} \\ p, p^{\prime}=1}}^{2 j+1} U_{p, p^{\prime}} n_{i, p} n_{i, p^{\prime}}
$$

where the infinite Hubbard interaction amplitude $U_{p, p^{\prime}}=+\infty$ between $p$ and $p^{\prime}$ bands excludes sites with double and more occupations. It follows from the above considerations that this model has energy levels which coincide with the levels of $X X Z$ Heisenberg model, but that the degeneracy of the levels is different.

For vanishing density-density interaction $V=0$ the Hamiltonian (18) describes the infinite repulsion limit of the multi-band Hubbard model. Thus, according to (15) $\Delta=0$ and it has the energy levels of free fermionic model.

\section{Multi-band extension of $t-J$ model, which has affine quan- tum group symmetry}

In this section we consider Hamiltonians which have the same energy levels as $t-J$ model but have affine quantum group symmetry. Because each site in ordinary $t-J$ model has three states, one should for this purpose take direct sum of three spaces. Let

$$
\mathbf{V}=\mathbf{V}_{0} \oplus \mathbf{V}_{j} \oplus \mathbf{V}_{j}
$$

Recall the $t-J$ model is given by

$$
\mathcal{H}_{t-J}(t, J, V)=\mathcal{P} \sum_{i=1}^{L-1}\left[-t \sum_{\sigma= \pm \frac{1}{2}}\left(c_{i, \sigma}^{+} c_{i+1, \sigma}+c_{i+1, \sigma}^{+} c_{i, \sigma}\right)+J \mathbf{S}_{i} \mathbf{S}_{i+1}+V n_{i} n_{i+1}\right] \mathcal{P}
$$

where $c_{\sigma}^{+}, c_{\sigma}$ are creation-annihilation operators of spin- $\frac{1}{2}$ fermion, $\mathbf{S}=\sum_{\sigma, \sigma^{\prime}} c_{\sigma}^{+} \sigma_{\sigma \sigma^{\prime}} c_{\sigma^{\prime}}$ is the fermionic spin operator and $\mathcal{P}=\prod_{i=1}^{L}\left(1-n_{i, \uparrow} n_{i, \downarrow}\right)$ forbids double occupation of sites.

We rewrite it in terms of Hubbard operators $X_{b}^{a}$, where $a, b=0, \pm \frac{1}{2}$ :

$$
\begin{aligned}
\mathcal{H}(t, J, V)=\sum_{i=1}^{L-1}\left[\sum _ { \sigma = \pm \frac { 1 } { 2 } } \left(-t\left(X_{i 0}^{\sigma} X_{i+1}^{0}+\right.\right.\right. & \left.\left.X_{i+10}^{\sigma} X_{i \sigma}^{0}\right)+\frac{1}{2} J \cdot X_{i-\sigma}^{\sigma} X_{i+1 \frac{\sigma}{\sigma}}\right) \\
& \left.+\sum_{\sigma, \sigma^{\prime}= \pm \frac{1}{2}}\left(\sigma \sigma^{\prime} J+V\right) X_{i \sigma}^{\sigma} X_{i+1} \underset{\sigma^{\prime}}{\sigma^{\prime}}\right]
\end{aligned}
$$


Let us now look at the general expression (4) of intertwining operators $H_{i j}$ acting on the space (19). For convenience we make index change in the following way. The two spin- $j$ representations we use are denoted by $\sigma= \pm \frac{1}{2}$. The intrinsic index in each $V_{j}^{(\sigma)}$ is denoted by $k, k=1, \ldots, 2 j+1$. So, instead of $\left(n_{i}, a_{i}\right)$ in (四) we have $(\sigma, k)$, if $i$ corresponds to spin- $j$ multiplet. Because the spin-0 singlet is one dimensional and single, we just use for it the index 0 . The non-equivalent irreps in $(\sqrt{19})$ are $V_{j}^{(\sigma)}$ and $V_{0}$ and, as mentioned above, the $R$-matrix for two such representations is the identity. After performing the first sum in (4) over non-equivalent multiplets we obtain

$$
\begin{array}{r}
H\left(A, a, b_{1}, b_{2}\right)=\sum_{\sigma_{1}, \sigma_{2}, \sigma_{1}^{\prime}, \sigma_{2}^{\prime}}\left(A_{\sigma_{1} \sigma_{1}^{\prime}}^{\sigma_{2} \sigma_{k, k^{\prime}}^{\prime}} X_{\left(\sigma_{2}, k\right)}^{\left(\sigma_{1}, k\right)} \otimes X_{\left(\sigma_{2}^{\prime}, k^{\prime}\right)}^{\left(\sigma^{\prime}, k^{\prime}\right)}\right)+a \cdot X_{0}^{0} \otimes X_{0}^{0} \\
+\sum_{k, \sigma}\left(b_{1} \cdot X_{0}^{(\sigma, k)} \otimes X_{(\sigma, k)}^{0}+b_{2} \cdot X_{(\sigma, k)}^{0} \otimes X_{0}^{(\sigma, k)}\right)
\end{array}
$$

To implement the restriction $H_{0}\left(A, a, b_{1}, b_{2}\right)$ of this operator on the highest weight space one just should eliminate the sum over $k, k^{\prime}$ and put $k=k^{\prime}=0$. Comparing (22) and (21) it follows that the expressions coincide if one chooses

$$
a=0 \quad b_{1}=b_{2}=-t \quad A_{\sigma-\sigma}^{-\sigma \sigma}=J / 2 \quad A_{\sigma \sigma}^{\sigma^{\prime} \sigma^{\prime}}=\left(\sigma \sigma^{\prime}\right) \cdot J+V
$$

and choose the other values of $A_{\sigma_{1} \sigma_{2}}^{\sigma_{1}^{\prime} \sigma_{2}^{\prime}}$ equal zero.

So, the Hamiltonian $\mathcal{H}\left(A, a, b_{1}, b_{2}\right)$ corresponding to (22) with these values of parameters gives rise to a $t-J$ model $(20)$ on the highest weight space. According to the previous considerations it will have the same energy levels as $t-J$ model, but with different degeneracy. Recall that for $J=2 t$ the $t-J$ model is "supersymmetric" and integrable.

We express the Hubbard operators in terms of multi-band fermionic creation-annihilation operators as follows

$$
\begin{array}{cc}
X_{i 0}^{(\sigma, k)}=\mathcal{P} c_{i, \sigma}^{k+}, & X_{i(\sigma, k)}^{0}=c_{i, \sigma}^{k} \mathcal{P}, \\
X_{i(-\sigma, k)}^{(\sigma, k)}=c_{i, \sigma}^{k+} c_{i,-\sigma}^{k} \mathcal{P}=\mathcal{P} c_{i, \sigma}^{k+} c_{i,-\sigma}^{k}, & X_{i(\sigma, k)}^{(\sigma, k)}=n_{i, \sigma}^{k} \mathcal{P}=\mathcal{P} n_{i, \sigma}^{k}
\end{array}
$$

Here as before we used the projection operator, which forbids double occupation on all sites

$$
\mathcal{P}=\prod_{i=1}^{L} \mathcal{P}_{i}, \quad \mathcal{P}_{i}=\prod_{(\sigma, k) \neq\left(\sigma^{\prime}, k^{\prime}\right)}\left(1-n_{i, \sigma}^{k} n_{i, \sigma^{\prime}}^{k^{\prime}}\right)
$$

Now, we can write down the Hamiltonian (5) in terms of multi-band fermions, substituting (23) into (22). We obtain in this way the multi-band generalization of (20)

$$
\mathcal{H}(t, J, V)=\mathcal{P} \sum_{i=1}^{L-1}\left[-t \sum_{k=1}^{2 j+1} \sum_{\sigma= \pm \frac{1}{2}}\left(c_{i, \sigma}^{k+} c_{i+1, \sigma}^{k}+c_{i+1, \sigma}^{k+} c_{i, \sigma}^{k}\right)+J \mathbf{S}_{i} \mathbf{S}_{i+1}+V n_{i} n_{i+1}\right] \mathcal{P}
$$

Here $k$ is the band index, and $\mathbf{S}=\sum_{k} \mathbf{S}^{k}, n=\sum_{k} n^{k}$ are total spin and total particle number operators. 


\section{References}

[1] T.Hakobyan and A.Sedrakyan. Phys. Lett., B377:250, 1996.

[2] V.Rittenberg, F.Alcaraz, D.Arnaudon and M.Scheunert. Int. J. Mod. Phys., A9:19, 1994.

[3] A. Avakyan, T.Hakobyan and A.Sedrakyan. Family of affine quantum group invariant integrable extensions of Hubbard Hamiltonian. Preprint YerPhI-1476(13)-96, condmat/9609183, 1996, Nucl. Phys. B, in press.

[4] C.N.Yang. Rev. Mod. Phys., 34:694, 1962.

[5] C.N.Yang and S.Zhang. Mod. Phys. Lett., B4:759, 1990.

[6] R.Strack. Phys. Rev. Lett., 70:833, 1993.

[7] A.A.Ovchinnikov. Mod. Phys. Lett., B7:1397, 1993.

[8] V.Korepin, J. de Boer and A.Schadschneider. Phys. Rev. Lett., 74:789, 1995.

[9] A.Montorsi and D.K.Campbell. Phys. Rev., B53:5153, 1996

[10] A.Schadschneider. Phys. Rev., B51:10386, 1995.

[11] P. W.Anderson. Science, 235:1196, 1987.

[12] F.C.Zhang and T.M.Rice. Phys. Rev., B37:3759, 1988.

[13] V. G.Drinfeld. Quantum groups. ICM proceedings, pages 798-820, 1986.

[14] M. Jimbo. Lett. Math. Phys., 10:63, 1985.

[15] M. Jimbo. Lett. Math. Phys., 11:247, 1986.

[16] V.Chari and A.Pressley. Commun. Math. Phys., 142:261, 1991. 